\title{
Microsaccades enable efficient synchrony-based visual feature learning and detection
}

\author{
Timothée Masquelier ${ }^{12^{*}}$, Geoffrey Portelli ${ }^{3}$, Pierre Kornprobst ${ }^{3}$ \\ From The Twenty Third Annual Computational Neuroscience Meeting: CNS*2014 \\ Québec City, Canada. 26-31 July 2014
}

Fixational eye movements are common across vertebrates, yet their functional roles, if any, are debated [1]. To investigate this issue, we exposed the Virtual Retina simulator [2] to natural images, generated realistic drifts and microsaccades using the model of ref. [3], and analyzed the output spike trains of the parvocellular retinal ganglion cells (RGC).

We first computed cross-correlograms between pairs of RGC that are strongly excited by the image corresponding to the mean eye position. Not surprisingly, in the absence of eye movements, that is when analyzing the tonic (sustained) response to a static image, these cross-correlograms are flat. Adding some slow drift $(\sim 20$ $\mathrm{min} / \mathrm{s}$, self-avoiding random walk) creates long timescale $(>1 \mathrm{~s})$ correlations because both cells tend to have high firing rates for central positions. Adding microsaccades $\left(\sim 0.5^{\circ}\right.$ in $25 \mathrm{~ms}$, that is $\left.\sim 20^{\circ} / \mathrm{s}\right)$ creates short timescale (tens of ms) correlations: cells that are strongly excited at a particular landing location tend to spike synchronously shortly after the landing.

What do the patterns of synchronous spikes represent? To investigate this issue, we fed the RGC spike trains to neurons equipped with spike timing-dependent plasticity (STDP) and lateral inhibitory connections, as in ref. [4]. Neurons self-organized, and each one selected a set of afferents that consistently fired synchronously. We then reconstructed the corresponding visual stimuli by convolving the synaptic weight matrices with the RGC receptive fields. In most cases, we could easily recognize what was learned (e.g. a face), and the neuron was selective (e.g. only responded for microsaccades that landed on a face). Without eye movements, or with only the drift, the STDP-based learning failed, because it

* Correspondence: timothee.masquelier@alum.mit.edu

${ }^{1}$ Institut de la Vision, UPMC Université Paris 06, Paris, 75012, France

Full list of author information is available at the end of the article needs correlations at a timescale roughly matching the STDP time constants [5].

Microsaccades are thus necessary to generate a synchrony-based coding scheme. More specifically, after each microsaccade landing, cells that are strongly excited by the image corresponding to the landing location tend to fire their first spikes synchronously. Patterns of synchronous spikes can be decoded rapidly - as soon as the first spikes are received - by downstream "coincidence detector" neurons, which do not need to know the landing times. Finally, the required connectivity to do so can spontaneously emerge with STDP. As a whole, these results suggest a new role for microsaccades - to enable efficient visual feature learning and detection thanks to synchronization - that differs from other proposals such as time-to-first spike coding with respect to microsaccade landing times.

\section{Acknowledgements \\ We thank A. Wohrer for having developed the Virtual Retina simulator and for the quality of his support, as well as M. Gilson for insightful discussions. The research received (partial) financial support from the 7th Framework Program for Research of the European Commission, under Grant agreement no 600847: RENVISION project of the Future and Emerging Technologies (FET) program (Neuro-bio-inspired systems (NBIS) FET-Proactive Initiative).}

\section{Authors' details}

'Institut de la Vision, UPMC Université Paris 06, Paris, 75012, France. ${ }^{2}$ CNRS, UMR 7210, Paris, 75012, France. ${ }^{3}$ Neuromathcomp Project Team, Inria Sophia Antipolis Méditerranée, 06902, France.

Published: 21 July 2014

\footnotetext{
References

1. Martinez-Conde S, Otero-Millan J, Macknik SL: The impact of microsaccades on vision: towards a unified theory of saccadic function. Nat Rev Neurosci 2013, 14:83-96.

2. Wohrer $A$, Kornprobst $P$ : Virtual Retina: a biological retina model and simulator, with contrast gain control. J Comput Neurosci 2009, 26:219-249.

3. Engbert R, Mergenthaler K, Sinn P, Pikovsky A: An integrated model of fixational eye movements and microsaccades. Proc Natl Acad Sci U S A 2011, 108:E765-70.

4. Masquelier T, Guyonneau R, Thorpe SJ: Competitive STDP-Based Spike Pattern Learning. Neural Comput 2009, 21:1259-1276.
} 
5. Gilson M, Masquelier T, Hugues E: STDP allows fast rate-modulated coding with Poisson-like spike trains. PLoS Comput Biol 2011, 7:e1002231.

doi:10.1186/1471-2202-15-S1-P121

Cite this article as: Masquelier et al:: Microsaccades enable efficient

synchrony-based visual feature learning and detection. BMC

Neuroscience 2014 15(Suppl 1):P121.

Submit your next manuscript to BioMed Central and take full advantage of:

- Convenient online submission

- Thorough peer review

- No space constraints or color figure charges

- Immediate publication on acceptance

- Inclusion in PubMed, CAS, Scopus and Google Scholar

- Research which is freely available for redistribution

Submit your manuscript at 